\title{
The Role of Task Representation in Completing an Integrated L2 Writing Task: Evidence from Eye-Tracking and Stimulated Recall
}

\author{
Pucheng Wang1, Baoquan Liu ${ }^{2 *}$, Hao Wang1 \\ ${ }^{1}$ School of Applied Foreign Languages, Zhejiang International Studies University, Hangzhou, China \\ ${ }^{2}$ School of Economics and Finance, Shanghai International Studies University, Shanghai, China \\ Email: p.wang@zisu.edu.cn, ^baoquanliuchina@163.com
}

How to cite this paper: Wang, P. C., Liu, B. Q., \& Wang, H. (2020). The Role of Task Representation in Completing an Integrated L2 Writing Task: Evidence from Eye-Tracking and Stimulated Recall. Open Journal of Modern Linguistics, 10, 773-784. https://doi.org/10.4236/ojml.2020.106047

Received: October 19, 2020

Accepted: November 28, 2020

Published: December 1, 2020

Copyright ( 2020 by author(s) and Scientific Research Publishing Inc. This work is licensed under the Creative Commons Attribution International License (CC BY 4.0).

http://creativecommons.org/licenses/by/4.0/

\begin{abstract}
Task representation is an essential step for second/foreign language writers when they compose either classroom writing assignments or write in a test situation. This study used a combination of eye-tracking and stimulated recall techniques to investigate the task representation processes of 16 non-native English writers who completed an integrated reading-to-write task. Results showed that the participants engaged in task representation processes throughout task completion. Evidence from their eye movements and stimulated verbal recalls proved that this type of cognitive process is not a single, simple act, but an extended, repetitive interpretive process that may occur at different phases of writing. The inclusion of reading materials in a writing task may complicate writers' task representation process by introducing more reading into the process of writing, and thus calls for more interaction between these two skills.
\end{abstract}

\section{Keywords}

Second Language Writing Assessment, Composing Process, Integrated Writing Tasks, Task Representation

\section{Introduction}

The past two decades have witnessed a growing interest among second/foreign language teachers and testers in integrated writing tasks. It is generally considered that writing is unlikely to be done separately from other language skills; instead, it tends to be dependent on gathering information from outside sources (Esmaeili, 2002; Hinkel, 2006; Hirvela, 2004). Compared with independent writing-only tasks, which have often been criticised for decontextualising writing 
activities and under-representing the writing construct, integrated writing tasks have been proposed as a promising task type in language teaching and assessment (Hamp-Lyons \& Kroll, 1997; Plakans, 2008; Weigle, 2004). For example, the reformed Test of English as a Foreign Language (TOEFL), which previously contained only a single independent writing-only task, added an integrated writing task that requires test-takers to listen to and read texts followed by a written summarisation based on this content.

However, a need exists for the field to improve our understanding of what second/foreign language writers do when faced with writing tasks that require integration of different skills, so as to inform language teachers of the impact of choosing between the two writing task types and to help guide use of the tasks for classroom teaching practices (Plakans, 2010). An essential step for writers composing for either type of writing tasks is to construct an understanding of the task demands. This construction of meaning has been labeled task representation in Flower, Stein, Ackerman, Kantz, McCormick and Peck's 1990 study, and defined as an interpretative process during which writers create a representation of the task by reading through the task instruction, which contains information about the topic of the task, rhetorical functions expected, for example, describing and discussing, and contextual constraints such as time constraints and word length, and sometimes scoring criteria and information about the input texts (in a reading-to-write task). This study examined foreign language writers' task representation processes while completing an English reading-to-write task, aiming to gain further insights into this particular type of cognitive processing on integrated writing tasks.

\section{Literature Review}

Task representation is an important process because students' performance is dependent on their understanding of the task. As discussed earlier in Hayes' (1996) writing model, if writers create a task representation based on a misunderstanding of the instructions, they may not be able to address the task appropriately. This process has been studied with first language (L1) writers (e.g., Flower et al., 1990) as well as second/foreign (L2/FL) language writers (e.g., Allen, 2004; Ruiz-Funes, 2001; Wolfersberger, 2007).

Flower et al. (1990) found that undergraduate students created different representations for the same reading-to-write task in terms of main sources of ideas, text features, organizational structure of the text, and strategies to use. Also, their results indicated that students with more experience in academic writing tended to create a more accurate task representation than students with less academic writing experience. Ruiz-Funes (2001) examined the written products of 14 Spanish-as-a-foreign-language students who composed an essay discussing a literacy text, and found that writers approached the task differently, and resulted in various rhetorical styles. The more cognitively complex style, however, did not lead to a text with more syntactically complex structures. Allen (2004) followed an English-as-a-second-language student through a linguistic class as- 
signment, finding that the student's representation of the task was greatly impacted by her prior experience in writing from external source materials. Similar to Allen's study, Wolfersberger (2007) found that four Chinese writers' representations of a classroom-based reading-to-write task were shaped by a variety of personal and contextual factors such as writers' background, prior experience, and interactions with course lecturers during the writing process. Chan (2013) developed and validated a reading-to-write process questionnaire and used it to investigate 219 students' cognitive processes while completing four reading-to-write tasks. The results of exploratory factor analysis confirmed the underlying construct of different cognitive processes that she proposed as core processes in a reading-to-write task. It was also found that higher-scoring students reported more use of task representation processes than lower-scoring students.

Some other language teachers or researchers have focused on comparisons of writers' use of task representation processes in completing independent and integrated writing tasks, and findings suggested that integrated reading-to-write tasks tap into a different set of literacy skills which go beyond those normally required by traditional independent writing tasks (Chan, Wu, \& Weir, 2014; Chan, 2017; Plakans, 2010). Plakans (2010) compared ten writers' task representation process in completing an integrated reading-to-write task and an independent writing task through think-aloud protocols and interview, finding that some writers failed to spot the difference between these two types of tasks, and used the same independent writing process to compose essays. Also, her findings revealed that all writers followed "an initial circular process of reading and rereading the integrated prompt that consumed time and increased the complexity of understanding the instructions in task" (Plakans, 2010: p. 193), which was not found in the independent writing task.

Among these studies that attempt to investigate the task representation process, the majority of them have focused on its impact on writers' written products (e.g., Ruiz-Funes, 2001), relatively few studies have been conducted to examine how these processes are employed during task completion, which is of particular interest given the focus of this study on use of this type of cognitive process. Among these few attempts to explore how writers approach an integrated writing task, most studies (e.g., Plakans, 2010) used think-aloud protocols, interview or questionnaire to collect and analyse data, each of which is considered problematic when used alone.

Therefore, in order to address the gap in research, it was decided to combine eye-tracking and stimulated recall methods to examine writers' online task representation processes while completing a reading-to-write task. This study contributes to the further understanding of this type of cognitive processes, and the usefulness of combining different research methods in integrated writing process studies is presented. Two research questions were proposed:

RQ1. How do writers approach an integrated reading-to-write task?

RQ2. To what extent do writers employ task representation processes while 
completing an integrated reading-to-write task?

\section{Method}

\subsection{Participants}

A total of 20 students participated in this study. They were all native Chinese learners of English and, at the time of data collection, were enrolled in either Linguistics and English Language or Finance programmes at a university in North West England. Two of the 20 participants proved to be unsuitable for being eye-tracked through "scanpath" inspection (Holmqvist et al., 2011). Data were then collected from the remaining 18 participants who had been successfully screened for eye-tracking suitability. Out of the 18 participants, two participants' data were excluded due to insufficient accuracy (weighted gaze samples < $50 \%$; $50 \%$ means that at least one eye was found for the full recording) for further analyses. The final data set therefore included 16 participants: 11 were female $(69 \%)$ and five were male (31\%); their ages ranged from 21 to 28 years $($ Mode $=23 ;$ Mean $=22.6 ; S D=1.66)$.

14 participants sat the International English Language Testing System (IELTS) test within one and a half year before data collection, while the other two took the test two years earlier. Table 1 summarises their performance on IELTS overall and on Reading and Writing components, respectively. According to the Common European Framework of Reference for Languages (CEFR), these participants' proficiency levels were between B2 and C1.

\subsection{Equipment and Instrument}

The participants' eye movements were recorded using a screen-based binocular tracking eye-tracker: Tobii TX300 (Tobii AB, Sweden). A sample reading-to-write task of the Test for Business English Majors-Band 8 (TBEM-8) was used to elicit participants' task representation processes. The topic of the task concerned Steve Jobs' resignation from Apple, and the task contained a set of instructions and five source materials in the prompt. This task was displayed on the eye-tracker screen during the experiment. The task instructions and the first three source materials were presented down the left part of the screen and the other two source materials and the answer sheet (where participants wrote the essay) were presented on the right part of the screen. Each part of the task was fixed on the screen, thus no scrolling was required, which made it possible for the eye-tracker to calculate eye movement data within each individual area on the screen.

Table 1. Participants' IELTS test scores.

\begin{tabular}{ccccccc}
\hline $\begin{array}{c}\text { IELTS/IELTS } \\
\text { components }\end{array}$ & Mean & Median & Mode & $\begin{array}{c}\text { Standard } \\
\text { Deviation }\end{array}$ & Minimum & Maximum \\
\hline Overall & 7.16 & 7.00 & 7.50 & 0.35 & 6.50 & 7.50 \\
Reading & 8.00 & 8.00 & 8.50 & 0.58 & 7.00 & 9.00 \\
Writing & 6.25 & 6.00 & 6.00 & 0.55 & 5.50 & 7.00 \\
\hline
\end{tabular}




\subsection{Data Collection}

The data were collected over two sessions. During the first session (eye-tracking session), the participants completed the reading-to-write task while their eye movements were being recorded by the Tobii TX300 eye-tracker. This was immediately followed by the second session (stimulated recall session), during which the participants were asked to verbalise their thoughts during task completion, using their eye traces recorded in the first session as stimuli for retrospection. The stimulated recall session was conducted in the participants' first language, Mandarin Chinese, and was audio- and video-recorded.

\subsection{Data Analyses}

Before the data analysis, the eye-tracker screen was divided into seven areas of interest (AOIs) corresponding to the seven parts of the TBEM-8 reading-to-write task, which include the task instructions, the five source materials and the answer sheet where the participant typed the essay. Having identified AOIs, the eye-tracker software can analyse fixation data within each individual area. In order to answer the first research question, that is, how do writers approach an integrated reading-to-write task, a type of eye-tracking metrics-Time to first fixation, was calculated to measure how long it takes before a participant fixates on an AOI for the first time.

Also, heat-maps resulting from the recordings of the participants' eye traces were generated. In a heat-map, different colours are used to display the accumulated fixation duration on different locations in the image displayed on screen and thus can be used, for example, to measure the amount of time dedicated to a particular area of interest. Red usually indicates the longest fixation duration and green the least, with varying levels in between, and the area where participants do not look at remains transparent. Figure 1 shows an example of heat-map
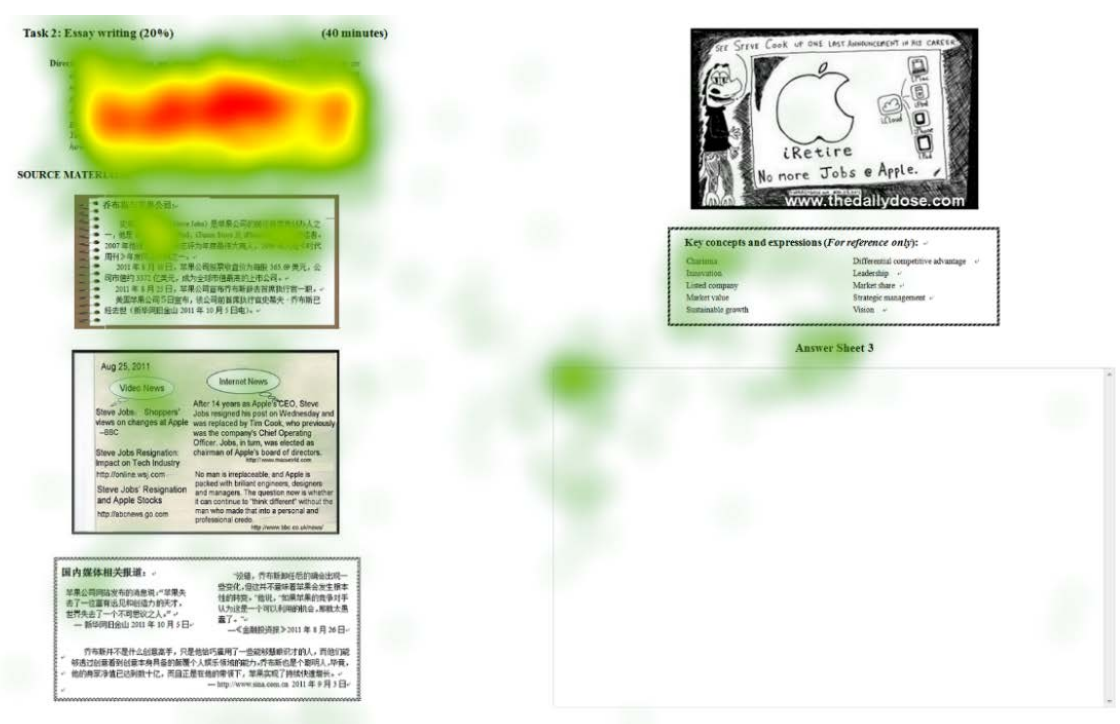

Figure 1. Heat-map of the TBEM-8 reading-to-write task for the first one minute of recording. 
visualisation. It can be seen in the figure that, during the first one minute of recording, the participants spent the majority of time on the area of instructions while other parts of the task received limited amounts of attention.

In order to answer the second research question, that is, to what extent do writers employ task representation processes while completing an integrated reading-to-write task, the participants' verbal reports were first transcribed by the researcher (a native Chinese speaker) based on the video recordings of the stimulated recall session. Then the transcriptions were coded to identify students' task representation processes throughout the task completion, and the number of occurrences for this type of cognitive processes at different stages of task completion was calculated. In addition, quotes from participants' stimulated recalls were presented to illustrate their use of task representation processes while completing the task.

\section{Results and Discussion}

\subsection{Eye-Tracking Metrics: Time to First Fixation}

Time to first fixation measures the amount of time from when an AOI was shown on the screen until the start of the first fixation within it. Table 2 shows the results on this metric by the 16 participants. The time-related metrics are all presented in seconds. It can be seen in the table that although participants approached the reading-to-write task quite differently in terms of the time when they looked at each AOI for the first time, a major pattern that seems to emerge from these measures (and by looking at participants' eye-movement recordings) is that participants started responding to the task by having a quick and short browse of all the seven parts of the task, and then went back to read the task instructions and the source materials one after another in a slow and careful manner. Figure 2 shows the heat-map output for the first 30 seconds of recording of Participant 6, which can be used as an example to illustrate this common pattern. During the first half minute, this participant's attention was scattered

Table 2. Time to first fixation on each individual AOI by participant.

\begin{tabular}{|c|c|c|c|c|c|c|c|c|c|c|c|c|c|c|c|c|c|c|}
\hline \multirow{3}{*}{$\begin{array}{c}\text { Areas } \\
\text { of interest }\end{array}$} & \multicolumn{18}{|c|}{ Participant } \\
\hline & $1(\mathrm{~F})$ & $2(\mathrm{~F})$ & $3(\mathrm{M})$ & $4(\mathrm{M})$ & $5(\mathrm{~F})$ & $6(\mathrm{M})$ & $7(\mathrm{~F})$ & $8(\mathrm{~F})$ & $9(\mathrm{~F})$ & $10(\mathrm{M})$ & $11(\mathrm{~F})$ & $12(\mathrm{~F})$ & $13(\mathrm{~F})$ & $14(\mathrm{~F})$ & $15(\mathrm{M})$ & $16(\mathrm{~F})$ & Min & $\operatorname{Max}$ \\
\hline & $\mathrm{Sec}$ & $\mathrm{Sec}$ & $\mathrm{Sec}$ & $\mathrm{Sec}$ & $\mathrm{Sec}$ & $\mathrm{Sec}$ & $\mathrm{Sec}$ & $\mathrm{Sec}$ & $\mathrm{Sec}$ & $\mathrm{Sec}$ & $\mathrm{Sec}$ & $\mathrm{Sec}$ & $\mathrm{Sec}$ & $\mathrm{Sec}$ & $\mathrm{Sec}$ & $\mathrm{Sec}$ & $\mathrm{Sec}$ & $\mathrm{Sec}$ \\
\hline Instructions & 0.9 & 97.2 & 0.6 & 1.1 & 1.3 & 4.0 & 0.6 & 1.5 & 5.3 & 5.1 & 3.8 & 5.6 & 1.1 & 1.8 & 0.8 & 3.9 & 0.6 & 97.2 \\
\hline Source 1 & 0.5 & 1.1 & 0.4 & 0.9 & 17.5 & 1.3 & 2.8 & 0.7 & 0.9 & 1.2 & 0.6 & 47.3 & 0.9 & 2.6 & 0.6 & 1.3 & 0.4 & 47.3 \\
\hline Source 2 & 3.5 & 0.7 & 128.4 & 0.6 & 15.5 & 2.0 & 135.5 & 45.1 & 31.6 & 11.6 & 2.4 & 3.5 & 76.4 & 1.1 & 60.8 & 2.4 & 0.6 & 135.5 \\
\hline Source 3 & 60.4 & 156.5 & 3.7 & 0.4 & 12.6 & 12.5 & 5.0 & 41.4 & 106.6 & 12.0 & 150.2 & 67.7 & 163.4 & 3.8 & 61.1 & 2.9 & 0.4 & 163.4 \\
\hline $\begin{array}{l}\text { Source } 4 \\
\text { (picture) }\end{array}$ & 181.3 & 490.8 & 1.5 & 147.1 & 10.1 & 3.5 & 209.3 & 37.7 & 136.1 & 1005.6 & 1.9 & 1.5 & 356.8 & 99.0 & 120.2 & 94.7 & 1.5 & 1005.6 \\
\hline Source 5 & 4.6 & 183.4 & 1.7 & 1.8 & 11.0 & 7.3 & 6.0 & 1.2 & 4.4 & 68.5 & 37.5 & 1.8 & 1.8 & 1.2 & 1.2 & 5.5 & 1.2 & 183.4 \\
\hline Answer sheet & 5.0 & 50.4 & 5.6 & 146.2 & 12.4 & 5.9 & 7.3 & 1.0 & 2.1 & 1.8 & 198.9 & 4.5 & 2.8 & 1.4 & 1.5 & 8.3 & 1.0 & 198.9 \\
\hline
\end{tabular}




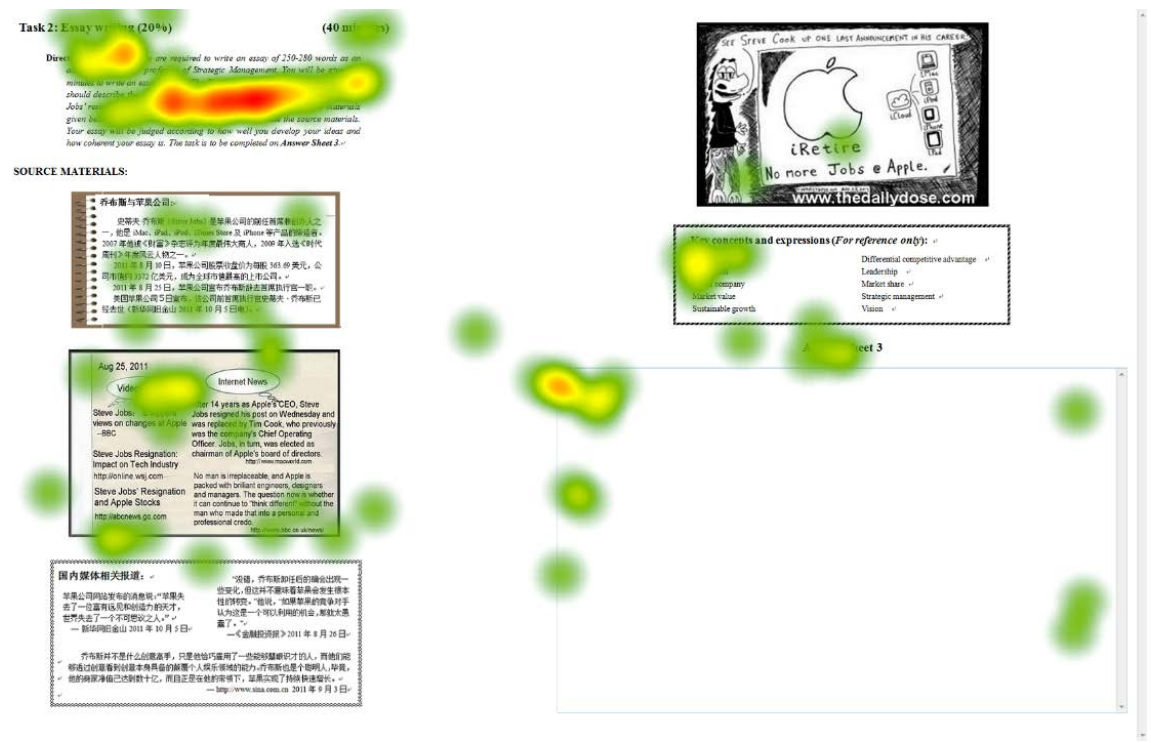

Figure 2. Heat map output for the first 30 seconds of recording of participant 6 .

loosely over different areas of the task, with a relatively strong focus on the task instructions. This is natural that at the beginning of the task completion test-takers may spend some time having a quick browse at each part of the task in order to get a general idea of what different parts are about, especially when they are not familiar with the task type. Participants then typically returned to read the instructions in detail to gain further understanding of the task.

There is only one participant, Participant 2, who did not follow this major pattern. Figure 3 shows how she approached the task in the first 90 seconds of her recording. Instead of reading the task instructions first, she started by moving straight to the first and second source texts, and the reading approach she adopted seemed to be more expeditious and local as her attention was unevenly spread within these materials. It was after these 90 seconds when she read the instructions for the first time and then went on reading through the other source materials. Also, it is interesting to note that it took Participant 10 nearly $17 \mathrm{mi}-$ nutes (maximum figure for Source 4) before he had the first fixation on Source 4 (the picture) and it seems that this material needs, on average, more time to attract participants' attention as compared to other source materials.

\subsection{Task Representation: Stimulated Recall Results}

The whole process of completing the TBEM-8 reading-to-write task was, for ease of analysis, divided into three phases: before writing, during writing and after writing. "Before writing" refers to the period during which participants get themselves ready for the writing process. In practice, this period was considered to start from the time at which participants' first fixation appeared on the eye-tracker screen and to end at the moment they typed the first word on the answer sheet. "During writing" is the major phase in task completion when participants compose a first draft of the essay, during which they are expected to 

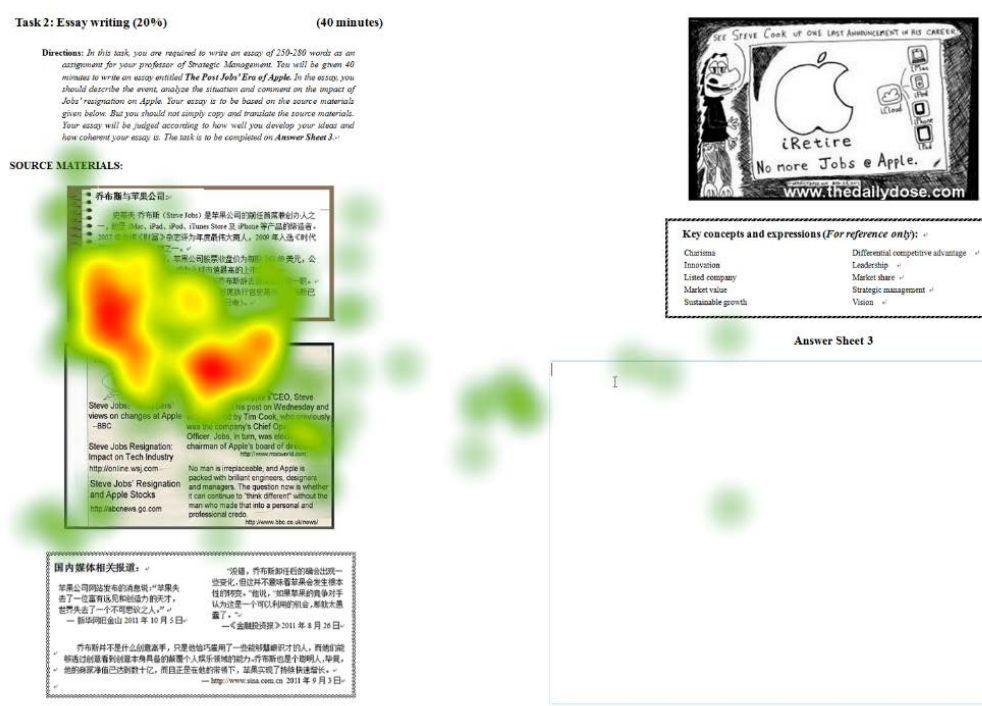

Figure 3. Heat map output for the first 90 seconds of recording of participant 2.

integrate information from the source materials into their writing and translate thoughts into words. Finally, the "after writing" phase is when participants finish the first draft and make revisions to their writing.

The 16 participants' verbal reports were parsed to differentiate the use of task representation process between different writing phases (see Table 3). The results show that the most instances of task representation occurred before participants started to write, fewer instances were found during writing and only four participants reported that they revisited the instructions after completing the first draft.

Before writing, with only one exception (see Figure 3), the participants started by reading through the instructions a first time to construct an initial understanding of the task demands, for example, Participant 6 read the instructions carefully, word by word, instantly after he had a quick browse of each part of the task at the beginning, “...I was reading the instructions, because they are very important, the title of the essay and task requirements were provided, I read closely this part...". Following this first reading, participants then moved on to read the source materials, during which they were found, through their eye-traces, going back to read the instructions a second or even more time. Participant 11 explained her circular process of reading and rereading the instructions:

I went on to read the following materials and went back to reread the instructions and then I understood what this task wanted us to write, especially about how many specific parts we should cover in the essay, for example, analysis of the situation and comments on Job's resignation.

During writing, most of the participants devoted less time to task representation than they did before starting to write. Two major patterns emerged when examining the protocols at this phase. First, participants revisited the task instructions for support on text they were about to produce. For example, Participant 
Table 3. Task representation at different phases of writing by participant.

\begin{tabular}{ccccccccccccccccccc}
\hline \multirow{2}{*}{ Writing phase } & Participant & \multicolumn{1}{c}{ 1 } & 2 & 3 & 4 & 5 & 6 & 7 & 8 & 9 & 10 & 11 & 12 & 13 & 14 & 15 & 16 & Total \\
\hline Before writing & 3 & 4 & 4 & 3 & 2 & 3 & 2 & 3 & 1 & 5 & 4 & 2 & 1 & 1 & 1 & 6 & 45 \\
During writing & 7 & 1 & 1 & 3 & 1 & 2 & 2 & 3 & 0 & 3 & 0 & 4 & 0 & 3 & 0 & 2 & 32 \\
After writing & 1 & 0 & 0 & 1 & 0 & 0 & 0 & 0 & 1 & 0 & 0 & 0 & 1 & 0 & 0 & 0 & 4 \\
$\quad$ Total & 11 & 5 & 5 & 7 & 3 & 5 & 4 & 6 & 2 & 8 & 4 & 6 & 2 & 4 & 1 & 8 & 81 \\
\hline
\end{tabular}

1 recalled, “...I was wondering what to write in the first sentence, then I went to read the first source material and the instructions, the instructions said that you should describe the event, so I thought I needed to describe it...". Second, the participants went back to the instructions to check if they went off the topic, for example, Participant 12 explained, “... when writing essays in Chinese, I always remind myself to stay on the topic. I worried about deviating from the topic, I thought it would be a serious problem, so I looked back to the instructions time and time again". Four participants reported that they engaged in the task representation process after they finished the first draft. Participant 4 was one of them: "now I had done checking the grammar, tense, vocabulary etc., then I went back to look at the instructions again to check if I missed any points."

To sum up, the participants used task representation processes during all phases of writing, especially before writing, when they read through the instructions for an initial understanding of the task demands, and during writing, when they revisited the instructions for either guidance on the text to be produced or monitoring the progress of their writing. When they looked back to the instructions, they seemed to spare relatively little time for the contextual constraints of the task such as input length and time limit, while they paid most of their attention to the content demands, i.e., what content is expected in the written product, either before writing, during writing or after writing.

Their protocols also indicated that the use of source materials in integrated writing tasks may complicate writers' task representation process, which agrees with findings in other research (Plakans, 2010; Wolfersberger, 2007). Participants in this study provided several reasons for their effort to understand the task. First, they reported that they had little experience with writing from source materials in a test setting, for example, Participant 2 commented on her recursion in the task representation process, "...then I read the materials and looked back to the instructions sometimes, because I have not done this kind of task before, so I needed to go back to reconsider its requirements". Second, due to the extra cognitive load of reading the source materials, their working memory seemed not capable to hold the content of the task instructions, so they tended to forget the information in the instructions. For instance, Participant 14 reported "...After reading (the source materials), I forgot what I was required to do, and then I went back there (the instructions) and checked it out again". 
Lastly, although some participants claimed that the source materials were helpful for understanding the task, for example, as described earlier, Participant 11 seemed not able to construct an accurate task representation in her first reading of the task instructions, but reading the source texts facilitated her understanding of it. However, there are other participants who thought the inclusion of source materials made their conceptualization of the topic even more complicated, for example, Participant 12 stated, “...After reading the source materials I became confused about what the task wanted me to write", then she returned to the instructions and reread the source materials several times for clarification, which makes her task representation process rather complex. This may be due to the participants' reading proficiency in that if they had trouble building a representation of the source materials or the instructions, they were less likely to comprehend the relationship between the materials and task, and thus hindered their task representation and other relevant processes, for example, macro-planning.

\section{Conclusion}

This study investigated foreign language writers' task representation processes while completing an integrated writing task. Findings from eye-tracking and stimulated recall reveal that, to complete the TBEM-8 reading-to-write task, the majority of students read the task instructions carefully to create an initial understanding of the task before writing, which aligns with previous research on this process (Allen, 2004; Chan, 2013; Flower et al., 1990). However, it was also found in this study that the task representation process occurred both during and after writing, proving that this process is not a single, simple act, but an extended, repetitive interpretive process throughout the task completion. Another finding was that the use of source materials in an integrated writing task may, to some extent, complicate writers' task representation process. This may be because the extra cognitive load involved in reading the source materials taxed writers' working memory to the extent that they may not have been capable of holding the content of task instructions in mind.

These findings have implications for the use of integrated writing tasks in both classroom and assessment settings. In classroom instruction, teachers should realise that students might not be familiar with this task type and may not properly understand the task demands and approach the task in the expected way. Students should be guided to gain a better understanding of the task requirements, including, but not limited to, comprehending the input materials, selecting and synthesising information, and integrating reading with writing. In a test situation, when writing instructions for such tasks, simple directions that clearly describe the purpose of the task and, in particular, what is expected to do with the source materials are ideal. However, as many studies have demonstrated, even with carefully constructed instructions, writers' task representation processes may differ; therefore, when interpreting the results from these tasks, it 
should be considered that writers may not have recognised tasks as required.

\section{Conflicts of Interest}

The authors declare no conflicts of interest regarding the publication of this paper.

\section{References}

Allen, S. (2004). Task Representation of a Japanese L2 Writing and Its Impact on the Usage of Source Text Information. Journal of Asian Pacific Communication, 14, 77-89. https://doi.org/10.1075/japc.14.1.06all

Chan, S. H. C. (2013). Establishing the Validity of Reading-into-Writing Test Tasks for the UK Academic Context. Unpublished PhD Thesis, Bedfordshire: The University of Bedfordshire.

Chan, S. H. C. (2017). Using Keystroke Logging to Understand Writers' Processes on a Reading-into-Writing Test. Language Testing in Asia, 7, 10.

https://doi.org/10.1186/s40468-017-0040-5

Chan, S. H. C., Wu, R. Y. F., \& Weir, C. J. (2014). Examining the Context and Cognitive Validity of the GEPT Advanced Writing Task 1: A Comparison with Real-Life Academic Writing Tasks (pp. 1-91). LTTC-GEPT Research Report 3.

Esmaeili, H. (2002). Integrated Reading and Writing Tasks and ESL Students' Reading and Writing Performance in an English Language Test. The Canadian Modern Language Review, 58, 599-622. https://doi.org/10.3138/cmlr.58.4.599

Flower, L., Stein, V., Ackerman, J., Kantz, M. J., McCormick, K., \& Peck, W. C. (1990). Reading-to-Write: Exploring a Cognitive and Social Process. New York: Oxford University Press.

Hamp-Lyons, L., \& Kroll, B. (1997). TOEFL 2000: Writing: Composition, Community and Assessment. Princeton, NJ: Educational Testing Service.

Hayes, J. R. (1996). A New Framework for Understanding Cognition and Affect in Writing. In C. M. Levy, \& S. Ransdell (Eds.), The Science of Writing: Theories, Methods, Individual Differences, and Applications (pp. 1-27). Mahwah, NJ: Lawrence Erlbaum Associates.

Hinkel, E. (2006). Current Perspectives on Teaching the Four Skills. TESOL Quarterly, 40, 109-131. https://doi.org/10.2307/40264513

Hirvela, A. (2004). Connecting Reading and Writing in Second Language Writing Instruction. Ann Arbor, MI: The University of Michigan Press.

https://doi.org/10.3998/mpub.23736

Holmqvist, K., Nystrom, M., Andersson, R., Dewhurst, R., Jarodzka, H., \& Van deWeijer, J. (2011). Eye Tracking. A Comprehensive Guide to Methods and Measures. Oxford: Oxford University Press.

Plakans, L. (2008). Comparing Composing Processes in Writing-Only and Reading-to-Write Test Tasks. Assessing Writing, 13, 79-150.

https://doi.org/10.1016/j.asw.2008.07.001

Plakans, L. (2010). Independent versus Integrated Writing Tasks: A Comparison of Task Representation. TESOL Quarterly, 44, 185-194. https://doi.org/10.5054/tq.2010.215251

Ruiz-Funes, M. (2001). Task Representation in Foreign Language Reading-to-Write. Foreign Language Annals, 34, 226-234. https://doi.org/10.1111/j.1944-9720.2001.tb02404.x

Weigle, S. C. (2004). Integrating Reading and Writing in a Competency Test for 
Non-Native Speakers of English. Assessing Writing, 9, 27-55.

https://doi.org/10.1016/j.asw.2004.01.002

Wolfersberger, M. A. (2007). Second Language Writing from Sources: An Ethnographic Study of an Argument Essay Task. Unpublished Doctoral Dissertation, Auckland: University of Auckland. 